\title{
TAGUNG
}

\section{Die Rolle der Europäischen Union im globalen Ordnungssystem}

\author{
Thomas Krück und Jens Senger*
}

Peter-Christian Müller-Graff wies einleitend auf die hohe Aktualität der Tagungsthematik hin. Der Vertrag von Lissabon sehe die Förderung der Weltordnung ausdrücklich als ein Ziel des auswärtigen Handelns der Union vor und auch der vor kurzem gewählte neue Präsident der USA Barack Obama habe im Rahmen seiner Rede in Berlin die Weltordnungspolitik thematisiert. Die Europäische Union habe aus Anlass der Wahl Obamas erklärt, dass sie eine neue globale Weltordnungspolitik anstrebe, welche die großen Herausforderungen des 21. Jahrhunderts wie Versorgungssicherheit mit Lebensmitteln, Klimawandel, Sicherheit, Energie, Rohstoffe, Finanzmärkte und Entwicklung einschließe. In der globalisierten Welt ergebe sich eine Vielzahl von Aufgaben für die Europäische Union, welche für sie eine Herausforderung und gleichzeitig auch eine große Chance darstellen. Müller-Graff betonte, dass die Tagung zum einen einer Bestandsaufnahme dienen und zum anderen das Potenzial der Europäischen Union als Akteur der Weltordnungspolitik erhellen solle.

\section{Weltordnungspolitische Einflussnahme durch die Europäische Union}

Werner Link erläuterte die Rolle der Europäischen Union in der Weltpolitik. Er verwies zunächst auf die in jüngerer Zeit gemachten weltordnungspolitischen Erfahrungen im Rahmen der Georgienkrise. Link stellte die Welt als dreistufiges Bezugsmodell dar und referierte über die grundlegende Weltord-

\section{Die Europäische Union als Akteur der Weltordnungspolitik}

Jahreskolloquium des Arbeitskreises

Europäische Integration e.V. in Verbindung mit dem Europäischen Graduiertenkolleg

„Systemtransformation und Wirtschaftsintegration im zusammenwachsenden Europa" mit Unterstützung der Europäischen Kommission

Heidelberg, 6./7. November 2008

Einführung: Weltordnungspolitik als Herausforderung der Europäischen Union

Prof. Dr. Dres. h.c. Peter-Christian MÜLLERGRAFF, Vorsitzender des Vorstandes des AEI, Universität Heidelberg

Die Europäische Union als Akteur der Weltordnungspolitik

Prof. Dr. Werner LINK, Universität zu Köln

Der Beitrag der Europäischen Union zu einer regionalen und globalen Sicherheitsarchitektur Dr. Andreas MAURER, Stiftung Wissenschaft und Politik, Berlin

Die Rolle der Europäischen Union bei der Gewährleistung der Bedingungen des Welthandels

Dr. Christoph HERRMANN, Universität München

Die Sicherheit der Versorgung mit Rohstoffen aus der Sicht der Europäischen Union

Dr. Colin VANCE, Rheinisch-Westfälisches Institut für Wirtschaftsforschung, Essen

Die Mitverantwortung der Europäischen Union im Bereich der globalen Klimaentwicklung Dr. Andreas LÖSCHEL, Zentrum für Europäische Wirtschaftsforschung, Mannheim

* Thomas Krück, Wissenschaftlicher Mitarbeiter, Institut für deutsches und europäisches Gesellschafts- und Wirtschaftsrecht, Universität Heidelberg.

Jens Senger, Wissenschaftlicher Mitarbeiter, Institut für deutsches und europäisches Gesellschafts- und Wirtschaftsrecht, Universität Heidelberg. 
nungsproblematik. Die Weltordnungspolitik sei konträr zur innerstaatlichen Ordnung der einzelnen Staaten zu sehen. Den hierbei evidenten Unterschied konstatierte Link in dem für einen Staat typischen Über- und Unterordnungsverhältnis zu seinen Bürgern, wogegen die Weltordnungspolitik durch den Gedanken der souveränen Gleichheit der Staaten geprägt sei. Aus diesem Gegensatz ergebe sich, dass auf weltpolitischer Ebene keine übergeordnete Instanz zur effektiven Durchsetzung völkerrechtlicher Verpflichtungen bestehe. Folge sei ein Macht- und Sicherheitsdilemma. Gerade in der Reduktion dieses Dilemmas liege eine prinzipielle Aufgabe, welcher sich die Weltordnungspolitik stellen müsse. Wesentlich hierbei sei eine ,Balance of Powers' zur Verhinderung einer Übermachtstellung eines staatlichen Akteurs.

Im Folgenden befasste sich Link mit der Rolle der Europäischen Union innerhalb der weltordnungspolitischen Struktur und stellte hierzu drei Thesen auf: Nach seiner ersten These bestehe ein Wille der Europäischen Union, zu einem mitentscheidenden Akteur in der Weltordnungspolitik zu werden. Das Ziel der Europäischen Union sei hierbei eine multipolare und multilaterale Weltordnung mit der Union in der Spitzengruppe. Link zog innerhalb dieser These einen Vergleich zur Sicherheitspolitik der USA und hob die Konkurrenz zwischen dem europäischen und atlantischen Sicherheitsrahmen hervor. Die Weltordnungspolitik der Europäischen Union sei gerade durch eine antihegemoniale und antiimperiale Handlungsweise geprägt. Die zweite von Link aufgestellte These bezog sich darauf, dass die Weltordnungspolitik der Europäischen Union das Problem der ,Zweigleisigkeit' zu meistern habe. Diese Zweigleisigkeit definierte Link dahingehend, dass die Mitgliedstaaten der Europäischen Union gleichzeitig auch Mitglieder in anderen weltordnungspolitisch bedeutsamen Organisationen seien. Gerade im Rahmen der aktuell bestehenden Finanzmarktkrise müsse ein Auftragshandeln der Europäischen Union durch ihre Führungsstaaten erfolgen. Durch
Die Finanzmarktkrise und ihre Folgen für Regulierung und Aufsicht

Dr. Steffen KERN, Deutsche Bank Research, Frankfurt a. M.

Europäische Zuwanderungspolitik: Chancen und Risiken aus europarechtlicher Sicht Prof. Dr. Dres. h.c. Peter-Christian MÜLLERGRAFF, Universität Heidelberg

Menschenwürde, Menschenrechte, Freiheit, Demokratie, Gleichheit und Rechtsstaatlichkeit als außenpolitische Agenda der Europäischen Union

Prof. Dr. Marianne KNEUER, Universität Eichstätt-Ingolstadt

Weltordnungspolitik der Europäischen Union zwischen völkerrechtlichen Verträgen und internationaler Organisationsbildung - unter Einbeziehung von Nachbarschaftspolitik und Mittelmeerunion als Formen privilegierter Partnerschaft

PD Dr. Carsten NOWAK, Universität Dresden

die Finanzmarktkrise habe sich eine neue weltordnungspolitische Machtverteilung ergeben, da sich die USA von einem Gläubigerzu einem Schuldnerstaat entwickelt hätten. Die Europäische Union müsse deshalb die Rolle eines mitgestaltenden Akteurs der Weltordnungspolitik übernehmen. Die dritte These Links bezog sich auf die beachtliche Breite des Spektrums der Beiträge der Europäischen Union zur Weltordnungspolitik. Link nannte hier den durch die Europäische Union geschaffenen Gleichgewichtsfaktor, die Währungsunion, ihre Welthandelspolitik und die Beeinflussung der Weltfinanzordnung. Sie habe im Weiteren zur globalen Konfliktregulierung beigetragen. Der wichtigste Beitrag der Europäischen Union sei jedoch in dem von ihr durchgeführten kooperativen Interregionalismus $\mathrm{zu}$ sehen, welcher $\mathrm{zu}$ einer gleichberechtigten Einbeziehung der Entwicklungsländer in die Weltordnungspolitik führe. Abschließend konstatierte Link die Positivität der Europäischen Union als Akteur der Weltordnungspolitik und verwies auf die Natur der Weltordnung als eine nicht statische, sondern bewegliche Ordnung. 
Kompetenzen und Rolle der EU in der Außenund Welthandelspolitik

Andreas Maurer befasste sich mit dem Beitrag der Europäischen Union zur Schaffung einer sowohl regionalen als auch globalen $\mathrm{Si}$ cherheitsarchitektur. Er stellte zunächst die dieses Thema betreffenden Änderungen durch den Reformvertrag von Lissabon dar, infolge derer das Amt des Hohen Vertreters der Union für die Außen- und Sicherheitspolitik mit der Funktion eines Vizepräsidenten der Europäischen Kommission verknüpft wird. Hieraus ergebe sich ein Spannungsverhältnis, da dieser Hohe Vertreter nicht nur für die Gemeinsame Außen- und Sicherheitspolitik, sondern für alle auswärtigen Politiken der Europäischen Union zuständig sei. Maurer stellte die aktuellen Trends der Außen- und Sicherheitspolitik der Europäischen Union dar: Die Sicherheitsarchitektur im engeren Sinne bestehe vor allem in der von der Europäischen Union durchgeführten Erweiterung ihrer selbst und in ihrer Nachbarschaftspolitik. Die Gemeinsame Außen- und Sicherheitspolitik und die Europäische Sicherheits- und Verteidigungspolitik seien meist nur mandatiert und ließen gerade kein proaktives und präventives Handeln zu. Gerade im Rahmen der Gemeinsamen Außen- und Sicherheitspolitik sei eine zunehmende Wahllosigkeit und Instrumentalisierung festzustellen. Sie sei vor allem durch die parallelen Außenpolitiken der einzelnen Mitgliedstaaten gefährdet. Im Weiteren sei eine immer weiter fortschreitende Militarisierung der Europäischen Union dadurch festzustellen, dass vermehrt militärische Entscheidungsträger in den Entscheidungsprozess eingebunden würden. Zudem werde durch die Zusammenfassung einer wachsenden Zahl von Bereichen unter das Kohärenzgebot dieses überstrapaziert, wobei gerade das Europäische Parlament versuche, sich hierbei zu profilieren. Maurer verwies anschließend auf die Defizite der bestehenden vertraglichen Regelungen, auf das Sicherheitspaket II, auf die Erklärungen 13 und 14 zum Lissabonner Vertrag zur Gemeinsamen Außen- und Sicherheitspolitik und auf die durch das Einstimmigkeitserfordernis bedingte drohende Handlungsunfähigkeit der Europäischen Union.

Mit der Rolle der Europäischen Union bei der Gewährleistung der Bedingungen des Welthandels befasste sich Christoph Herrmann. Er betonte zunächst die sich hierbei ergebenden grundlegenden Fragestellungen nach den Kompetenzen der Europäischen Union für die Wirtschaftspolitik und den Zielvorgaben des Primärrechts hinsichtlich dieser Kompetenzen. Darauf folgend nahm Herrmann eine Definition des Begriffs, Gewährleistung der Bedingungen des Welthandels ' dahingehend vor, dass hierunter die Sicherung der Märkte und das Wirken gegen das Handelshemmnis der divergierenden technischen Standards der einzelnen Akteure des Welthandels und gegen das Wiederaufleben protektionistischer Verhaltensweisen zu verstehen sei. Insgesamt sei diese Gewährleistung als eine multidimensionale Balanceübung zu verstehen. Herrmann erläuterte nunmehr eingehend die zu Beginn formulierte erste Frage hinsichtlich der Kompetenzen der Europäischen Union zur Gewährleistung der Bedingungen des Welthandels. Er betonte zunächst das Prinzip der begrenzten Einzelermächtigung und verwies als einschlägige Kompetenzgrundlage auf die Bestimmungen des Titel IX, Gemeinsame Handelspolitik, des Vertrages zur Gründung der Europäischen Gemeinschaft (EGV). Er schenkte vor allem der ausschließlichen AuBenvertretungskompetenz der Europäischen Union in der Außenhandelspolitik gemäß Artikel 133 EGV Beachtung, kritisierte diese jedoch als missglückt und unlesbar. Als Kompetenzgrundlagen außerhalb des Titels IX des EGV verwies Herrmann auf Artikel $111 \mathrm{EGV}$ hinsichtlich der Außenwirkung der Eurozone und auf Artikel 174 EGV hinsichtlich der Umweltpolitik. Insgesamt bewertete Herrmann das momentane Kompetenzgefüge des EGV als unvollständig, wobei es zusätzlich große Abgrenzungsschwierigkeiten in sich berge. Im Folgenden wurde der zweiten eingangs formulierten Frage hinsichtlich der Zielvorgaben des Primärrechts der Europä- 
ischen Union für die Gewährleistung der Bedingungen des Welthandels nachgegangen. Herrmann betonte, dass solch klare Zielvorgaben - abgesehen vom Bereich des Umweltschutzes - fehlen und sich gerade auch nicht aus Artikel 2 des Vertrags über die Europäische Union (EUV) ergäben. Die Europäische Union habe insgesamt vielgestaltige Zielvorgaben, jedoch gerade keine speziellen auf dem Gebiet des Welthandels. Sodann widmete sich Herrmann den wesentlichen Änderungen infolge des Vertrags von Lissabon. Hierdurch werde die Europäische Union mit eigener Rechtspersönlichkeit ausgestattet und in den Katalog, welcher ihre Ziele bestimme, werden die nachhaltige Entwicklung und die Integration aller Länder in die Weltwirtschaft aufgenommen. Ein wesentliches Defizit im Rahmen dieses Katalogs sei das Fehlen von Kollisions- und Vorrangregelungen, was zu parallelen Zuständigkeiten führe. Als Beispiele für die internationale Wirtschaftspolitik der Europäischen Union in der jüngeren Vergangenheit wurden ihr Beitrag in der DohaRunde und die Reform der handelspolitischen Schutzinstrumente beschrieben. Im Rahmen einer Schlussbemerkung betonte Herrmann die Komplexität des Welthandels, die insgesamt durch den Vertrag von Lissabon angestrebte positive Entwicklung der Europäischen Union und ihre immer bedeutender werdende Rolle im Bereich des Welthandels.

\section{Rohstoffsicherung und ökologische Verant- wortung der Europäischen Union}

Colin Vance befasste sich mit der Gewährleistung der Versorgung mit Rohstoffen aus dem Blickfeld der Europäischen Union. Am Beispiel von Zink und Kupfer, welche aber auch repräsentativ für andere Rohstoffe seien, stellte Vance den tendenziell weltweit steigenden Rohstoffverbrauch dar, dessen Ursache die immer größer werdende Nachfrage in den sich zu Industrieländern entwickelnden Schwellenländern sei. Vor allem China verbrauche heutzutage in vielen Bereichen mehr Rohstoffe als die USA. Darüber hinaus sei es nur eine Frage der Zeit, bis der Rohstoffhun- ger Indiens erwache. Zukünftig werde die Nachfrage nach Rohstoffen wegen der zunehmenden weltweiten Industrialisierung immer weiter ansteigen. Der immer stärker werdenden Nachfrage nach Rohstoffen stellte Vance die Entwicklung der Rohstoffpreise gegenüber: Die Rohstoffpreise hätten sich in den vergangenen Jahren zwar stark erhöht, eine weitergehende Preissteigerung sei jedoch gerade nicht zu erwarten. Vance illustrierte am Beispiel von Kupfer und Aluminium, dass, real betrachtet, viele Rohstoffe mit der Zeit sogar günstiger werden. Bei allen Unsicherheiten über die künftige Rohstoffpreisentwicklung ergeben sich die Gewissheiten, dass zum einen die Rohstoffmärkte und -preise Zyklen aufwiesen und dass die Rohstoffpreise in der Regel zeitverzögert der weltweiten wirtschaftlichen Entwicklung folgten. Im Folgenden ging Vance auf die Problematik der Reserven, Ressourcen und Reichweiten von Rohstoffen ein: Es sei eine unumstößliche Tatsache, dass die Rohstoffvorkommen auf der Erde endlich seien. Allerdings sei für keine Rohstoffart eine absolute Knappheit beziehungsweise die Erschöpfung der Rohstoffvorräte erkennbar. Dies sei auch dadurch bedingt, dass durch Recycling große Rohstoffmengen wiedergewonnen werden können. So seien etwa Aluminium und Kupfer theoretisch zu 100 Prozent recyclebar. Als nächsten Punkt sprach Vance die regionalen Unterschiede in Verbrauch und Förderung der Rohstoffe an. Viele Rohstoffe befinden sich gerade nicht an dem Ort, an dem sie benötigt werden. Am Beispiel von Kupfer und Zink wurde dargestellt, dass derzeit China und die USA die größten Verbraucher dieser Rohstoffe seien, das bedeutendste Kupferförderland jedoch Chile sei und Zink am stärksten von China, Australien und Peru gefördert werde. In Deutschland etwa werde Zink seit 1992 überhaupt nicht mehr gefördert, obwohl die Bundesrepublik an fünfter Position der globalen Zinkverbraucher stehe. Zusammenfassend und abschließend stellte Vance fest, dass die Nachfrage nach Rohstoffen und der Handel mit Rohstoffen immer weiter zunehme, die Endlichkeit der Rohstoffvorkom- 
men hierbei jedoch keine Grenze für das globale Wirtschaftswachstum darstelle. Real betrachtet seien in der Vergangenheit die große Mehrheit aller Rohstoffe tendenziell sogar günstiger geworden, wobei aber auch zukünftig eine Rohstoffpreisschwankung zu erwarten sei.

Andreas Löschel stellte die globale Klimaentwicklung und die Mitverantwortung der Europäischen Union für diese dar. Er gab zunächst einen Überblick über den derzeitigen Stand des Klimawandels. Es sei ein weltweiter Temperaturanstieg zu verzeichnen, dessen Ursache sich auf menschliches Verhalten zurückführen lasse, namentlich auf den steigenden Ausstoß von Kohlenstoffdioxid. Die Rolle des Staates zur Verhinderung eines weiteren Temperaturanstiegs bestehe nunmehr darin, durch die Instrumente des Emissionshandels oder einer Steuer eine Lenkungswirkung auf die Wirtschaftsakteure auszuüben. Löschel betonte jedoch die Problematik einer solchen staatlichen Intervention, die unter anderem in dem Schadenspotenzial von Anpassungsmaßnahmen, ihrer räumlichen und zeitlichen Disparität und in der Kostenunsicherheit läge. Als Lösungsmöglichkeiten zur Reduktion des globalen Kohlenstoffdioxidausstoßes wurde auf die globale Klimapolitik, vor allem auf die Einhaltung der Kyoto-Ziele, verwiesen, wobei auch hier Unsicherheiten über deren Auswirkungen und Effizienz bestünden. Danach befasste sich Löschel im Besonderen mit der Klimapolitik der Europäischen Union. Er verwies hierbei auf die Umsetzung des Kyoto-Protokolls innerhalb der Europäischen Union, ihren Emissionshandel und ihre Handelsmaßnahmen. Im Rahmen des Gipfels der Europäischen Union im Jahre 2007 seien die Ziele ihrer Klimapolitik, vor allem die Senkung des Kohlenstoffdioxidausstoßes, bis zum Jahr 2020 beschlossen worden. Hierbei seien auch die Kosten, die Auswirkungen auf die Wettbewerbsfähigkeit und auf verschiedene Industrien sowie die Handelsintensität möglicher Maßnahmen zur Zielerreichung diskutiert worden. Abschließend bezeichnete Löschel die Klimapolitik als globale Heraus. forderung des 21. Jahrhunderts und betonte die Vorreiterrolle der Europäischen Union in diesem Bereich, wobei das Finden eines globalen Ansatzes von äußerster Wichtigkeit zur Lösung der Klimaproblematik sei.

\section{Aktuelle Fragen der Finanzmarktkrise}

Steffen Kern befasste sich mit der Finanzmarktkrise und ihren Folgen. Er begann mit einem Überblick über die gegenwärtige wirtschaftliche Lage, wobei er zunächst auf Entstehung und Entwicklung der Finanzkrise zurückblickte. Aktuell sei eine leichte Entspannung am Geldmarkt zu erkennen, dennoch bestehe eine gewisse Nervosität fort. Die staatlichen Rettungspakte zeigten bereits ihre Auswirkungen, jedoch seien die Emissionsmärkte und die Kapitalmarktaktivität stark geschwächt. Vor allem hohe Verluste auf Seiten der Banken sowie drastische Kursverluste an den Börsen seien zu beklagen. Insgesamt zeige sich die Realwirtschaft deutlich geschwächt. Daraufhin analysierte Kern die nationalen Rettungspakete und deren grundlegenden Elemente. Diese bestünden beispielsweise aus Liquiditätshilfen seitens der Zentralbanken durch die Übernahme der Geldmarktfunktion oder teilweise auch durch den direkten Erwerb der Unternehmensschulden. Zudem würden von der Krise betroffene Banken durch Verstaatlichung oder partielle staatliche Beteiligung rekapitalisiert und illiquide Aktiva übernommen. Jedoch stelle sich vor allem bei der staatlichen Beteiligung an Banken die Frage, ob diese zwangsweise erfolgen solle oder ob Überzeugungsarbeit geleistet werden müsse. Zum Schutz der Bankkunden vor drohenden Insolvenzen werde das Einlagensicherungssystem entweder durch eine Aufstockung des gesicherten Mindestbetrages oder durch umfassende staatliche Garantien verbessert. Laut Statistiken belaufe sich der Umfang der finanziellen Zusagen global auf 3,7 Billionen Euro. Im Weiteren widmete sich Kern der internationalen Koordinierung der Rettungsmaßnahmen, wobei er insbesondere auf den G7-Aktionsplan, den Weltfinanzgipfel der G20-Staaten und die hochrangige Experten- 
gruppe zur EU-Finanzaufsicht einging. Jedoch ließen die bislang unternommenen Maßnahmen sowohl auf nationaler als auch auf internationaler Ebene einige Punkte offen. So sei die Wirkung der Pakete in der Praxis noch nicht geklärt, ebenso wie die Frage, welche Methode zur Schaffung des Anreizes für die Marktteilnehmer, auf das Rettungsangebot einzugehen - Zwangskapitalisierung oder Rekapitalisierung auf freiwilliger Basis - effektiver sei. Auch in Bezug auf die internationale Koordinierung der Maßnahmen erwiesen sich noch einige Punkte als ungewiss, wie beispielsweise die Frage, ob die Grundprinzipien der Rettungsaktionen konkret genug oder ob die Regulierungs- und Aufsichtsstrukturen und -prozesse hinreichend koordiniert seien. Ebenso ungelöst sei auch die mögliche Einbindung der Finanzindustrie. Im dritten und letzten Teil seines Referats widmete sich Kern den Auswirkungen der Finanzmarktkrise auf die europäische Integration. Dabei erläuterte er sowohl unmittelbare Maßnahmen der Europäischen Union als auch längerfristige Reformen. So einigten sich beispielsweise die Länder der Eurozone auf einen abgestimmten Aktionsplan, um der Finanzkrise entgegenzusteuern. Zudem solle eine Zelle für Finanzkrisen geschaffen werden, um ein effektiveres Handeln in Krisensituationen zu gewährleisten, und makroökonomische Initiativen, wie ein Stabilitäts- und Wachstumspakt, ergriffen werden. Um dauerhaft die Auswirkungen der Finanzkrise zu beseitigen und weiteren Krisen vorzubeugen, sollen auf dem zweiten Weltfinanzgipfel der G20-Staaten am 2. April 2009 entsprechende Maßnahmen beschlossen werden. In der Europäischen Union seien hierfür eine Finanzmarktregulierung sowie die Optimierung der Aufsichtsstrukturen geplant. Laut Kern solle die Europäische Union im Bereich des weltweiten Krisenmanagements eine internationale Führungsrolle übernehmen.

Europäische Einwanderungspolitik - rechtlicher Rahmen und aktuelle Politik

Die Betrachtung der Außenpolitik der Europäischen Union im globalen Ordnungssystem er- öffnete Peter-Christian Müller-Graff mit seinem Vortrag zur europäischen Migrationspolitik. Einleitend stellte er anhand neuester Statistiken Europa als Zuwanderungsregion dar, bevor er sich der rechtlichen Dimension des Themas widmete und die Frage aufwarf, welche Rolle die Europäische Gemeinschaft in diesem Bereich spiele. Dabei beschäftigte sich Müller-Graff zunächst mit der Kompetenzlage der europäischen Zuwanderungspolitik. Die Verbandskompetenzen der Europäischen Gemeinschaft befänden sich in den Artikeln 61 bis 64 EGV und seien in systematischer Hinsicht dem Bereich des freien binnenmarktlichen Personenverkehrs zuzuordnen. Sie umfassten Maßnahmen bezüglich der Kontrolle der Außengrenzen der Mitgliedstaaten, die Asyl- und Flüchtlingspolitik sowie die Einwanderungspolitik. Hierbei handele es sich, wie Müller-Graff ausführte, nicht um ausschließliche, sondern um konkurrierende Gemeinschaftskompetenzen. Demzufolge verblieben Maßnahmen in diesen Politikbereichen in der Zuständigkeit der Mitgliedstaaten, soweit die Europäische Gemeinschaft nicht von ihren Kompetenzen Gebrauch mache. Darüber hinaus bestehe in Artikel 64 EGV eine Ordnungs- und Sicherheitsklausel zugunsten der Mitgliedstaaten. Im zweiten Teil seines Vortrags analysierte Müller-Graff den Sinngrund der migrationspolitischen Verbandskompetenzen der Europäischen Gemeinschaft. Als ursprüngliche Gründe für ihre Schaffung nannte er die Gefährdung der Funktionsfähigkeit des Binnenmarktsystems durch unterschiedliche Zuwanderungspolitiken der einzelnen Mitglieder. Der Raum ohne Binnengrenzen, der den freien Verkehr der Produktionsfaktoren gewährleisten solle, verlange somit eine koordinierte Migrationspolitik. Eine gemeinschaftliche Zuwanderungspolitik liege daher im gemeinsamen Interesse der Mitgliedstaaten. Dem sei dadurch Rechnung getragen worden, dass durch den Vertrag von Maastricht 1992 die flankierenden Maßnahmen zum freien Personenverkehr in die intergouvernementale dritte Säule der Europäischen Union aufgenommen worden seien, von wo sie durch den Vertrag von Amsterdam 
1997 in die supranationale erste Säule überführt wurden. Sodann warf Müller-Graff die Frage auf, ob nicht durch den Verfassungsvertrag und den Vertrag von Lissabon ein Paradigmenwechsel im Bereich der Migrationspolitik und der Integration erfolge. Dafür spräche deren vorgesehene Abkopplung vom Binnenmarktkonzept. Andererseits figurierten unter dem Titel „Raum der Freiheit, der Sicherheit und des Rechts" lediglich Ermächtigungen und keine unmittelbar anwendbaren Normen. Zudem sei anzumerken, dass drei Mitgliedstaaten (Großbritannien, Irland und Dänemark) an diesem Titel nur in begrenztem Umfang teilnehmen. Im Folgenden unterzog er die sekundärrechtlichen Regelungen im Bereich der Migrationspolitik einer eingehenden Betrachtung. So zeichne sich der Bereich der Visapolitik durch eine weitgehende Vergemeinschaftung aus. Insbesondere sei in diesem Zusammenhang der Schengen-Besitzstand zu erwähnen. Bezüglich der Asylpolitik bemerkte Müller-Graff, dass kein einheitliches System von Sekundärrechtssetzung vorliege. Allerdings sei das Dublin-Übereinkommen, nun abgelöst von der Dublin-II-Verordnung, diesbezüglich im Gemeinschaftsacquis hervorzuheben. Auf dem Gebiet der Einwanderungspolitik erwähnte er das Tampere-Programm, welches deren notwendige Bestandteile festlege. Jedoch sei sowohl bezüglich der legalen als auch der illegalen Einwanderung ein moderater, lediglich Einzelmaßnahmen umfassender Umsetzungsacquis zu erkennen. Aus der differenzierten Nutzung der gemeinschaftlichen Kompetenzen in den Bereichen Visa-, Asyl- und Einwanderungspolitik ließe sich, so Müller-Graff, der Rückschluss ziehen, dass in der Europäischen Gemeinschaft eine höhere Bereitschaft zur Abwehr von Zuwanderung als zur aktiven Gestattung bestehe. Die Gründe hierfür lägen vor allem in einer unterschiedlichen Auffassung der einzelnen Mitgliedstaaten bezüglich der Chancen und Risiken von Migration und Integration von Migranten. Müller-Graff beendete seinen Vortrag mit einigen zusammenfassenden Bemerkungen, die hervorhoben, dass Einwanderung und die Eingliederung von Einwanderern von hoher wirtschaftlicher Bedeutung für den Binnenmarkt seien und sich dadurch für die Europäische Gemeinschaft große Chancen ergäben. Einen letzten Blick warf er auf die aktuelle Lage und auf mögliche zukünftige Entwicklungen. Nachdem ein Vorstoß des französischen Staatspräsidenten und Vorsitzenden des Europäischen Rates im zweiten Halbjahr 2008 Nicolas Sarkozy, welcher eine festgeschriebene Kontingentierung der $\mathrm{Zu}$ wanderung vorsah, abgelehnt wurde, befinde sich nun ein Pakt zur Steuerung der Migration in der Diskussion.

\section{Europäische Demokratisierungspolitik - Konzept und Instrumente}

Einen Einblick in die europäische Demokratisierungspolitik gewährte Marianne Kneuer, indem sie sich mit der Förderung von Menschenrechten und Demokratie durch die Europäische Union befasste. Einleitend gab sie einen Überblick über die Entwicklung der europäischen Demokratisierungspolitik, in welchem sie erläuterte, wie sich historisch zunächst über die Beitrittskriterien - repräsentative Demokratie, Rechtsstaatlichkeit und Achtung der Menschenrechte zu zentralen Merkmalen der europäischen Außenpolitik entwickelt hätten. Im zweiten Teil ihres Vortrages erläuterte Kneuer, dass sich der Erweiterungsprozess der Europäischen Union als erfolgreiche Demokratisierungspolitik erweise. Dies sei vor allem darauf zurückzuführen, dass hierdurch ein signifikanter Einfluss der Europäischen Union auf die Beitrittskandidaten ausgeübt werde. Die in Aussicht gestellte Mitgliedschaft stelle sich als Hebel dar und das Konzept der Konditionalität der Beitrittsverhandlungen bei deren Aufnahme und Durchführung wirke als Anreiz-DruckSystem. Zu den Vorteilen der Erweiterungspolitik, die sich als Keimzelle der Demokratisierung erweise, gehöre trotz eines gewissen konzeptionellen und strategischen Defizits deren flexible Handhabung. Nach Ausführungen zu dem der Demokratisierungspolitik zugrunde liegenden Begriff von Demokratie, dessen Verständnis sich im Laufe der Zeit von 
einem minimalen zu einem sehr umfassenden ausweitete, widmete sich Kneuer dem Instrumentarium der Demokratisierungspolitik und ging insbesondere auf die Konditionalität als zentrale Methode ein. Diese erweise sich als ein Konzept sui generis, welches ein System von Anreizen für und Druck auf die Beitrittskandidaten, den von der Europäischen Union gestellten Kriterien bezüglich des Demokratisierungsprozesses zu genügen, darstelle. Fortschritt und Erfüllung der Bedingungen würden belohnt, während Defizite Sanktionen nach sich zögen. Der Erfolg der Konditionalität sei - so Kneuer - mit Blick auf die Südund Osterweiterung der Union unbestritten. Sie funktioniere auch in komplizierteren Fällen, wie in demjenigen der Slowakei. Die Bewerberstaaten würden bei ihrem Demokratisierungsprozess unterstützt, wobei sich die Europäische Union als konstanter Partner erweise. Die Referentin merkte jedoch auch an, dass dem System Grenzen gesetzt seien. Regierungen, welche keine Demokratisierung anstreben, seien kaum bereit, die Kriterien zu erfüllen. Zudem ginge der Hebel der in Aussicht gestellten Mitgliedschaft nach dem Beitritt verloren. Die vor der Erweiterung installierten Sicherheitsklauseln gälten nur für den Binnenmarktbereich, erlaubten aber keine demokratische Regression. Die einzig verbleibende Sanktionsmöglichkeit sei die Suspendierung der Mitgliedschaft nach Artikel 7 EUV, der jedoch noch nie angewandt wurde und dessen Voraussetzungen unklar seien. Zusammenfassend hielt Kneuer fest, dass die Demokratisierungspolitik der Europäischen Union kein festes Konzept sei, sondern sich dynamisch entwickle. Somit bestehe die Möglichkeit, auf neue Herausforderungen entsprechend zu reagieren. Vorzüge der europäischen Demokratieförderung seien neben ihrer Flexibilität ihr ziviler Charakter, welcher sie von der ,Demokratisierungspolitik ${ }^{6}$ der USA abhebe. Dennoch sehe sie sich mit einigen Problemen konfrontiert. So gebe es beispielsweise ein strategisches Defizit und einen Mangel an Kohärenz seitens der Europäischen Union. Auf der Seite der Drittländer wirke der Hebel der Konditionalität je nach
Ausprägung der Demokratisierungsbestrebungen unterschiedlich stark. Schließlich fordere der Umgang mit Autokratien völlig neue Konzepte.

Internationale Organisationen und Partnerschaften im Fokus der Außenpolitik der Europäischen Union

Zum Abschluss der Tagung widmete sich Carsten Nowak der Rolle der Europäischen Union in der Weltordnungspolitik am Beispiel von völkerrechtlichen Verträgen und der Bildung internationaler Organisationen, wobei er näher auf die Europäische Nachbarschaftspolitik und die Union für das Mittelmeer einging. Nach einigen einleitenden Worten zur Thematik der Tagung erläuterte Nowak, wie und inwiefern die Europäische Union durch völkerrechtliche Verträge und in internationalen Organisationen engagiert ist. So beteilige sich beispielsweise die Europäische Gemeinschaft auf globaler Ebene im Rahmen der Welthandelsorganisation und nach eigenen Angaben - in 35 weiteren internationalen Organisationen multilateral. Auch innerhalb der Vereinten Nationen wirke sie durch die Koordinierung der einzelnen Mitgliedstaaten mit. Im Rahmen der Außenbeziehungen sei - so Nowak - jedoch eine starke bilaterale Komponente vorhanden. Auf regionaler Ebene engagiere sich die Europäische Union in sogenannten Europäischen Partnerschaften. Nowak ging näher auf den Stabilisierungs- und Assoziierungsprozess Westlicher Balkan ein. Ziel des Prozesses sei es, durch Aktionspläne die Staaten Albanien, Bosnien und Herzegowina, Montenegro und Serbien an die Union anzunähern, für Stabilität in dieser Region zu sorgen und diesen Ländern eine Beitrittsperspektive zu eröffnen. Als Anreiz für die Umsetzung würden einzelne bilaterale Stabilisierungs- und Assoziierungsabkommen in Aussicht gestellt. Darüber hinaus existiere zwischen der Europäischen Gemeinschaft und den Staaten der Balkanregion die Energiegemeinschaft, welche eine Internationale Organisation zur Schaffung eines integrierten Energiemarktes sei. Insofern sei, 
wie Nowak anmerkte, ein komplementäres Verhältnis von völkerrechtlichen Verträgen und der Bildung Internationaler Organisationen zu erkennen. Dies stelle jedoch kein Novum dar, betrachte man die Parallelität des Ostseerates und der Europaabkommen mit den Mittel- und Osteuropäischen Staaten vor deren Beitritt zur Europäischen Union. Im nächsten Abschnitt seines Vortrages erörterte er die Verbindung bilateraler und multilateraler Arrangements am Beispiel der Europäischen Nachbarschaftspolitik und der Union für das Mittelmeer. Dabei führte er aus, dass es das Ziel der Nachbarschaftspolitik sei, einen Ring von stabilen und wohlhabenden Nachbarstaaten der Europäischen Union zu schaffen, welche deren Werte teilen, ohne jedoch eine Beitrittsperspektive zu haben. Die Union für das Mittelmeer - eine Gemeinschaft zwischen der Europäischen Union, den Mittelmeeranrainerstaaten sowie Mauretanien und Jordanien - habe zum Ziel, eine Partner- schaft für Zusammenarbeit auf gleicher Augenhöhe zu begründen. Es gebe also partielle Überschneidungen zwischen der Europäischen Nachbarschaftspolitik und der Union für das Mittelmeer. Nach Meinung Nowaks seien beide als privilegierte Partnerschaften zu begreifen, so dass auch hierbei von einer gewissen Parallelität gesprochen werden könne. Abschließend hielt Nowak fest, dass sich die Weltordnungspolitik der Europäischen Union sowohl durch den Abschluss völkerrechtlicher Verträge als auch durch die Bildung internationaler Organisationen manifestiere. In Bezug auf zukünftige Entwicklungen sei die Union für das Mittelmeer nicht als Schlusspunkt der internationalen Organisationsbildung zu betrachten. Vielmehr sei - ergänzend hierzu - eine ,Ost-Europa-Union“ unter Beteiligung von Staaten wie beispielsweise der Ukraine, Weißrusslands und der Republik Moldau denkbar.

\section{Externe Demokratisierung in Südosteuropa}

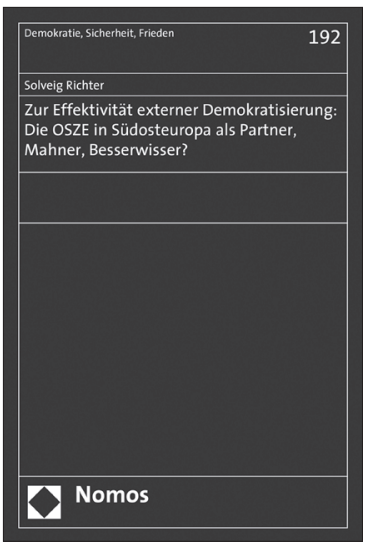

Bitte bestellen Sie im Buchhandel oder versandkostenfrei unter www.nomos-shop.de

\author{
Zur Effektivität externer \\ Demokratisierung: Die OSZE \\ in Südosteuropa als Partner, \\ Mahner, Besserwisser? \\ Von Dr. Solveig Richter \\ 2009, 453 S., brosch., 79,- $€$, \\ ISBN 978-3-8329-4378-3 \\ (Demokratie, Sicherheit, Frieden, Bd. 192)
}

